The Journal of Steroid Biochemistry and Molecular Biology

Special issue: Update on Steroids and Nervous System"

\title{
STRESS-INDUCED MECHANISMS IN MENTAL ILLNESS: A ROLE FOR GLUCOCORTICOID SIGNALLING
}

A. Cattaneo ${ }^{1,2}$ and M.A. Riva ${ }^{3}$

${ }^{1}$ Stress, Psychiatry and Immunology Laboratory, Department of Psychological Medicine, Institute of Psychiatry, King's College London, London, UK; ${ }^{2}$ IRCCS Fatebenefratelli San Giovanni di Dio, Brescia, Italy and ${ }^{3}$ Laboratory of Psychopharmacology and Molecular Psychiatry, Department of Pharmacological and Biomolecular Sciences, University of Milan, Via Balzaretti 9, Milan (Italy).

Corresponding author:

Prof. Marco Andrea Riva

Department of Pharmacological and Biomolecular Sciences, University of Milan, Via Balzaretti 9, Milan (Italy). Fax +39-02-50318278;

e-mail: M.Riva@unimi.it 


\section{Abstract}

Stress represents the main environmental risk factor for mental illness. Exposure to stressful events, particularly early in life, has been associated with increased incidence and susceptibility of major depressive disorders as well as of other psychiatric illnesses. Among the key players in these events are glucocorticoid receptors. Dysfunctional glucocorticoid signalling may indeed contribute to psychopathology through a number of mechanisms that regulate the response to acute or chronic stress and that affect the function of genes and systems known to be relevant for mood disorders. Indeed, exposure to chronic stress early in life as well as in adulthood has been shown to reduce the expression of glucocorticoid receptors (GR), also through epigenetic mechanisms, and to up-regulate the expression of the co-chaperone gene FKBP5, which restrains GR activity by limiting the translocation of the receptor complex to the nucleus. Another mechanism that contributes to changes in GR responsiveness is the state of receptor phosphorylation that controls activation, subcellular localization as well as its transcriptional activity. Moreover, GR phosphorylation may represent an important mechanism for the cross talk between neurotrophic signalling and GR-dependent transcription, bridging two important players for mood disorders. One gene that lies downstream from GR and may contribute to stress-related changes is serum glucocorticoid kinase-1 (SGK1). We have demonstrated that the expression of SGK1 is significantly increased after exposure to chronic stress in rodents as well as in the blood of drug-free depressed patients. We have also shown that SGK1 up-regulation may ultimately reduce hippocampal neurogenesis and contribute to the structural abnormalities that have been reported to occur in depressed patients.

In summary, GR signalling may represent a point of convergence as well as of divergence for defects associated with pathologic conditions characterized by heightened vulnerability to stress. The characterization of these abnormalities is crucial to identify novel targets for therapeutic intervention that may counteract more effectively stress-induced neurobiological abnormalities. 


\section{Stress and mental illness}

Stress represents a condition that implies a modification of homeostasis, which may occur through a number of different events regulating emotion, behaviour, cognition as well as physical health. While eustress has a positive meaning, distress originates from a persistency in stress exposure that is not associated with proper coping and may ultimately lead to pathologic consequences [1]. Indeed, stress represents the main environmental components for the susceptibility to mental illness, although it is known that the response to stress is modulated by the genetic signature as well as by a number of other factors, including earlier exposure to adverse life events that may have 'primed' the brain toward enhanced susceptibility. All these elements, and their reciprocal interaction, affect the ability to cope with stress thus leading to resilience or susceptibility. There are different ways through which stress may alter brain function and homeostasis, including the anatomical signature of the stress response, which include the regulation of specific pathways and circuits [2]. Another important aspect is the timing of stress occurrence and its implications for the functional outcome [3-9]. One critical time frame is represented by early life, which may span from prenatal to early postnatal life until adolescence. Taking into consideration the complex maturation profile of different brain structures and circuits, it may be inferred that exposure to stress or adverse life events can produce a wide array of changes with different functional consequences that may also depend upon the duration of the adverse experience [10]. Accordingly, it may be inferred that the psychopathologic consequences associated with stress exposure depend upon all these variables and their interaction with the genetic background.

While the stress response is determined by the integration of different mechanisms and mediators, in this review we will focus on altered glucocorticoid signalling as major contributor for long-term disability associated with stress-induced mental illness. While many peptides can be released under stressful conditions, within the brain, the action of corticosteroids is restricted by the presence of corticosteroid receptors, namely type I (mineralocorticoid receptors - MR) and type II (glucocorticoid receptors - GR), which are primarily ligand-activated transcription factors [11-13], but that may also operate through membrane receptors $[12,13]$. 


\section{Modulation of glucocorticoid receptors}

The glucocorticoid receptors are the major player in mediating many physiological events set in motion by stress exposure, including central as well as peripheral mechanisms with a pleiotropic response of the body's, including the modulation of metabolic pathways and immune-related mechanisms [12, 13]. Upon glucocorticoid binding, the receptor is released from a chaperone complex and translocates to the nucleus, where it regulates the transcription of target genes, by direct interaction with its response elements as well as through the modulation of transcription factors or other regulatory elements [14].

The function of the hypothalamus-pituitary-adrenal (HPA) axis is controlled by a feedback mechanism, which serves to terminate the 'acute stress response'. Indeed, endogenous glucocorticoids are potent negative regulators of the HPA axis activity by binding to their receptors in different tissues, including hypothalamus, pituitary as well as hippocampus.

On these bases, following exposure to stress or any condition that may alter body's homeostasis, there is a release of different mediators, including CRH and glucocorticoids (such as cortisol) that activate their receptors in different brain regions to determine a wide range changes, leading to behavioral, cognitive an functional changes that are supposed to encode for the proper coping response to stress. However, when this is not achieved or when stress continues, a wide range of modifications, including neuro-structural alterations as well as epigenomic changes will take place thus leading to pathologic consequences [2, 15].

A number of transgenic approaches in mice have demonstrated a causal role for glucocorticoid receptors in models for mood disorders, mimicking key features of depression and anxiety [16-20]. On the other end, exposure to stressful events early in life has been associated with increased incidence and susceptibility for depression as well as for other psychiatric illnesses $[3,6]$. It has been proposed that exposure to early life trauma may lead to a 'sensitization' of circuits and pathways that regulate emotion and stress responses and may ultimately lead to an enhanced vulnerability to subsequent stressors as well as to the development of depression and anxiety [21, 22]. This evidence has been corroborated by a number of studies in rodents that have investigated the mechanisms altered as a consequence of exposure to stressors or adverse conditions during gestation as well as during the first weeks of life. Pioneer studies by Meaney and coworkers have originally shown that offspring raised by 
dams with high levels of maternal care (high licking/grooming and arched-back nursing posture; high LG-ABN) are less fearful in exploring a novel environment, when compared to those raised by low LG-ABN [23], and show reduced HPA axis activation in response to an acute stress [24]. One mechanism that may be responsible for these effects is the reduced expression of GRs observed in the hippocampus of low LG-ABN, an effect that is sustained by an epigenetic mechanism [25]. DNA methylation is a covalent modification of the cytosine residues that are located primarily at $\mathrm{CPG}$ dinucleotide sequences: increased DNA methylation in the promoter regions is usually associated with repressed gene expression. Low LG-ABN rats show increased DNA methylation at a neuron-specific glucocorticoid receptor (NR3C1) promoter, which determines reduced expression of the receptor as well as increased HPA reactivity [25]. Interestingly, decreased levels of glucocorticoid receptor mRNA and increased cytosine methylation of a NR3C1 promoter were also found in the hippocampus obtained from suicide victims with a history of childhood abuse, as compared to suicide victims with no childhood abuse or controls [26]. These data provide translational relevance for the results obtained in rats and suggest a common effect of parental care on the epigenetic regulation of hippocampal glucocorticoid receptor expression.

Similar changes in the expression of GRs, as well as in the control of HPA axis function, were found using experimental paradigms that mimic exposure to adversities early in life, such as prenatal stress and maternal deprivation. For example, in the model of prenatal stress, consisting in exposing pregnant dams to daily stress sessions during the last week of gestation, we found a significant reduction in the expression of 11 beta-hydroxysteroid dehydrogenase- 2 (11B-HSD2) in the fetal part of the placenta (Riva et al., unpublished observations). These data are in agreement with previous results that have also shown reduced placental 11B-HSD2 mRNA levels following chronic restrain stress during gestation in rats, an effect that was accompanied with increase methylation at $3 \mathrm{CPG}$ sites in the promoter region of the 11B-HSD2 gene [27]. Considering the role of 11B-HSD2 in buffering maternal glucocorticoid by converting cortisol and corticosterone into inactive metabolites, its reduced expression may lead to a potential overload of glucocorticoids in the fetus that are suspected to impair normal development with long-term adverse consequences, such as dysregulation of the infant's HPA axis and neurobehavioral changes [28-30]. In agreement with this possibility, rats that have been exposed to stress in 
utero display increased anxiety and depression-like behavior, as well as enhanced stress sensitivity at adulthood, which is sustained by altered HPA axis activity and reduced expression of both GR and MR in the hippocampus [31, 32](Luoni et al., submitted). Withdrawal from maternal care also determines a persistent dysregulation of the HPA axis with decreased hippocampal GR levels and impaired feedback mechanisms [33-35]. Similar studies have been performed in non-human primates [36]. All these data suggest that changes in the expression of GRs may represent the first level of dysfunction in glucocorticoid signaling, leading to altered responsiveness to challenging conditions as well as behavioral alterations resembling mood disorders.

\section{Modulation of the chaperone protein FKPB5}

Another important player that may contribute to stress-related HPA dysfunction is the chaperone protein FKPB5 (FK506 binding protein 51)[12, 37]. FKBP5 represents an intracellular ultra-short negative feedback player that regulates glucocorticoid receptor activity. Indeed GR activation induces FKBP5 transcription, which may restrain glucocorticoid receptor activity by limiting the translocation of the receptor complex to the nucleus. The FKBP5 gene represents a good example for the interaction between genetic risk factor and exposure to environmental adversities in modulating the risk of developing major depressive disorders [38]. The FKBP5 gene is characterized by the presence of polymorphisms that have been associated with increased recurrence risk of depressive episodes and that may also moderate the risk for PTSD associated with childhood adversities [39]. The effect of the FKBP5 gene polymorphism to enhance the risk of developing stress-related psychiatric disorders in adulthood appears to be allele-specific and depends upon epigenetic changes in functional glucocorticoid response elements of FKBP5 as a consequence of childhood trauma exposure [40].

Changes in the expression of FKBP5 may also occur following exposure to stress in adulthood. We found that animals exposed to the paradigm of chronic mild stress (CMS), which may recapitulate depression-related disturbances, show increased expression of FKBP5 primarily in the ventral hippocampus and prefrontal cortex [41]. These changes are associated with enhanced levels of glucocorticoid receptors in the cytosolic compartment, which is suggestive of an impaired ability of the receptor to translocate to the nucleus and activate GR-dependent transcriptional 
mechanisms [41]. Interestingly, we have also demonstrated that chronic treatment with the serotonin-noradrenaline reuptake inhibitor duloxetine is able to reduce FKBP5 mRNA levels in the ventral hippocampus, and completely normalizes the changes of FKBP5 mRNA and protein levels found in the prefrontal cortex of CMS rats [41], suggesting that therapeutic intervention may restore the proper function of HPA axis activity by modulating the expression of the chaperone protein FKBP5. These results are in agreement with in vitro and in vivo studies, suggesting a close interaction between antidepressants and the GR system pointing to the idea that antidepressants enhance glucocorticoid sensitivity and function, may increase nuclear translocation of GRs and also facilitate GR-mediated gene transcription [42, 43].

\section{Modulation of glucocorticoid receptor phosphorylation}

Another mechanism that may contribute to GR responsiveness is the state of receptor phosphorylation, which controls activation, subcellular localization, gene transcription, and turnover of GRs [44, 45]. The receptor can be phosphorylated as a consequence of ligand binding as well as through the activation of different signaling pathways [45]. Hence GR phosphorylation represents a converging point for different intracellular mechanisms that co-operate in modulating GR function and responsiveness. We have shown that the phosphorylation of GR on Ser232, and to less extent on Ser224, is significantly reduced in the prefrontal cortex of rats that were exposed to the paradigm of CMS. It has been previously demonstrated that GR phosphorylation at these sites can regulate GR trafficking to the nucleus [42]. Hence, the reduced phosphorylation on Ser232 may represent another mechanisms through which stress exposure can impair GR function, by preventing or reducing its translocation into the nucleus. We have also demonstrated that phosphorylation on pSer224 and pSer232 is significantly upregulated by chronic duloxetine treatment, as previously shown to occur with sertraline in human hippocampal stem cells [42]. This effect normalizes the changes produced by CMS, particularly when considering pSer232 and may contribute to restore altered GR function and GR-dependent changes of HPA axis activity.

GR phosphorylation at Ser224 and Ser232 represents an important mechanism in the control of GR-dependent gene transcription by glucocorticoid hormones and antidepressants [42]. GR phosphorylation may also represent an important mechanism for the cross talk between 
neurotrophic signalling and GR-dependent transcription, bridging two important players for mood disorders. It is well established that the expression and function of trophic factors, such as brainderived neurotrophic factor (BDNF), is defective in selected brain regions of patients affected by stress-related disorders as well as in different animal models of depression [16, 46-52]. Lambert and coworkers have elegantly shown that the simultaneous treatment with BDNF and the GR agonist dexamethasone can produce a unique set of GR-responsive genes that play a role in neuronal growth and differentiation and may also enhance the transcription of a large number of dexamethasone-sensitive genes [53]. Interestingly, BDNF treatment induces the phosphorylation of GR at specific sites, a mechanism that may specify and amplify the GR transcriptome through coordinated GR phosphorylation. This observation may allow to speculate on the pathologic implications of the disruption of such mechanism in psychiatric disorders. Indeed, while one important consequence of the exposure to prolonged stressors can be glucocorticoid resistance due to the reduced ability of GR to activate, when required, the proper cellular responses, this situation can be aggravated by the dysfunction of other systems, such as the neurotrophin BDNF, that are important to promote neuronal resilience.

On these bases, it is important to investigate and characterize mechanisms downstream from GR activation that play a role in neuronal function within specific brain structures. One example is represented by the hippocampus that is strongly involved in the pathogenesis of depressive disorders. Indeed this brain structure is highly sensitive to stress [5] and it is one of the key regions identified as a component of a network that is altered in depressed patients $[54,55]$. It has been consistently demonstrated that the volume of the hippocampus is significantly smaller in patients with MDD, an effect that may be sustained by a number of different mechanisms including reduced trophic support as well as altered glucocorticoid sensitivity and responsiveness [15]. While cell atrophy has been reported to occur as a consequence of prolonged stress exposure, there is growing evidence that depression may be associated with reduced hippocampal neurogenesis that can ultimately contribute to decreased hippocampal volume [56, 57]. A large number of studies have shown that exposure to acute and chronic stress may affect neurogenesis, primarily cell proliferation, in several species [58, 59], an effect that is mainly mediated by stress hormones, such as glucocorticoids, which can regulate GR-dependent transcription in dividing cells [60]. Impaired hippocampal neurogenesis in rodents may contribute 
to the development of depressive symptoms in response to stress, supporting the notion that glucocorticoids-induced reduction in neurogenesis may indeed contribute to the pathogenesis of depression [57]. With this respect, we have recently shown that the glucocorticoid receptorregulated gene serum/glucocorticoid-regulated kinase 1 (SGK1), which is a target of GR, mediates the cortisol-induced decrease of neurogenesis in human hippocampal progenitor stem cells [61].

\section{Modulation of serum/glucocorticoid-regulated kinase 1}

SGK1 is a serine/threonine kinase implicated in the cellular response stress as well as in neuronal functions and it mediates some of the effects exerted by glucocorticoids on the brain. Following exposures to physiological stressors, the SGK1 gene is rapidly transcribed through the activation of GR and MR, and its protein undergoes constitutive phosphorylation via endogenous PI3-K activity [62]. The rapid transcriptional modulation of SGK1 levels represents one of the mechanisms through which glucocorticoids may activate several neuroendocrine responses [63, 64]. For example, in the rodent prefrontal cortex, acute stress up-regulates SGK1 that, on its turn, increases the trafficking and function of NMDARs and AMPARs, which leads to a potentiation of synaptic transmission, thereby facilitating cognitive processes mediated by prefrontal cortex activation [65]. Conversely, in the murine corpus callosum, chronic stress can cause morphological abnormalities in oligodendrocytes by up-regulating SGK1 [66]. We found that SGK1 is involved in the regulation of hippocampal neurogenesis and, in particular, may be responsible for the effects of cortisol on the proliferation and neuronal differentiation of human hippocampal progenitor cells [61]. This may occur through the inhibition of the Hedgehog pathway as well as through the regulation of glucocorticoid receptor phosphorylation and its nuclear translocation [61]. Indeed, when we treated human hippocampal progenitor cells with the stress hormone cortisol we observed a significant reduction of neurogenesis, an effect that was accompanied by increased levels of SGK1. Importantly, co-treatment of the cells with cortisol and GSK 650394, the small inhibitor of SGK1, was able to prevent the negative effect of cortisol on neurogenesis. In order to provide further support to the role of SGK1 in stress response, we investigated its expression in animals exposed to prolonged stress. We found that SGK1 mRNA levels are significantly increased in the dorsal and ventral hippocampus of rats 
exposed to chronic mild stress as well as in the hippocampus of adult rats that were exposed to stress during gestation [61]. We also found that SGK1 mRNA levels are significantly higher in the blood of drug-free depressed patients, thus providing support for the potential clinical implication of these findings. Interestingly, in agreement with our in vitro data showing decreased GR levels in the presence of increased SGK1 expression, SGK1 mRNA levels show a negative correlation with GR levels in our clinical sample. Moreover, SGK1 correlated positively with the mRNA levels for the GR target gene FKBP5 and negatively with the expression of the neurotrophin BDNF $[61,67]$. Recent findings have also shown that repeated stressful events can induce a sustained elevation of plasma corticosterone levels and an up-regulation of SGK1 mRNA expression in oligodendrocytes, causing excess arborization of oligodendrocyte processes that is thought to contribute to depressive-like symptoms [68]. Moreover, in line with our cellular work demonstrating a role for SGK1 in neurogenesis, it has been recently demonstrated that the expression levels of several GR inducible genes, including SGK1, correlated with lower hippocampal volumes, providing a potential link between glucocorticoid signalling, SGK1 and hippocampal volume abnormalities found in stress-related mental disorders [69, 70]. Based on these data, it may be inferred that SGK1 gene represents a fine sensor that contributes to acute and chronic stress responses in different brain structures. However, it must be borne in mind that SGK1 is only one example of the complex pattern of gene regulation that lies downstream from the activation of GR and may contribute to psychopathological mechanisms affected in mood disorders. Among these, we have extensively investigated the expression of neuroplastic genes, such as the neurotrophin BDNF, as well as inflammatory mediators, that show protracted modifications as a function of exposure to stressful experiences early in life as well as in adulthood $[3,7,71,72]$.

In summary, increasing evidence indicates that psychiatric disorders are characterized by complex changes in the function and responsiveness of GRs, which represent a point of convergence as well as of divergence for defects associated with pathologic conditions characterized by heightened vulnerability to stress. While, under acute conditions GR-dependent mechanisms may contribute to stress coping and resilience, following protracted exposure to adverse conditions a derangement of the signalling pathways downstream from GRs is observed. 
The characterization of these abnormalities is crucial to identify novel targets for therapeutic intervention that may counteract more effectively stress-induced neurobiological abnormalities. As an example, potent and highly selective inhibitors of FKBP51, SAFit1 and SAFit2, have been recently developed. This new class of ligands enhances neurite elongation in neuronal cultures and improves neuroendocrine feedback and stress-coping behavior in mice, suggesting that direct interference with FKBP5 function may hold promise as novel therapeutic intervention in stress-related disorders [73].

\section{Conflict of Interest}

The author A. Cattaneo has no financial interest or potential conflict of interest. Riva M.A. has received compensation as speaker/consultant as well as research support from Bristol-Myers Squibb, Sumitomo Dainippon Pharma, Eli Lilly, Innova Pharma, Lundbeck, Servier, Sunovion and Takeda.

\section{Acknowledgments}

This work was supported by grants from the Italian Ministry of University and Research to M.A.R. (Progetti di Ricerca di Interesse Nazionale - PRIN - grant number 20107MSMA4_002), from Fondazione CARIPLO (grant number 2012-0503) to M.A.R., from European Union/NEURON-ERANET to M.A.R. and from European Union/NEURON-ERANET to A.C. 


\section{Bibliography}

[1] H. Selye, A Syndrome produced by Diverse Nocuous Agents, Nature, 138 (1936) 32.

[2] B.S. McEwen, Physiology and neurobiology of stress and adaptation: central role of the brain, Physiol Rev, 87 (2007) 873-904.

[3] N. Provencal, E.B. Binder, The effects of early life stress on the epigenome: From the womb to adulthood and even before, Experimental neurology, 268 (2015) 10-20.

[4] E.R. de Kloet, R.M. Sibug, F.M. Helmerhorst, M. Schmidt, Stress, genes and the mechanism of programming the brain for later life, Neurosci Biobehav Rev, 29 (2005) 271-281.

[5] S.J. Lupien, B.S. McEwen, M.R. Gunnar, C. Heim, Effects of stress throughout the lifespan on the brain, behaviour and cognition, Nature reviews. Neuroscience, 10 (2009) 434-445.

[6] C. Heim, E.B. Binder, Current research trends in early life stress and depression: review of human studies on sensitive periods, gene-environment interactions, and epigenetics, Experimental neurology, 233 (2012) 102-111.

[7] A. Luoni, A. Berry, F. Calabrese, S. Capoccia, V. Bellisario, P. Gass, F. Cirulli, M.A. Riva, Delayed BDNF alterations in the prefrontal cortex of rats exposed to prenatal stress: preventive effect of lurasidone treatment during adolescence, European neuropsychopharmacology, 24 (2014) 986-995.

[8] A. Berry, P. Panetta, A. Luoni, V. Bellisario, S. Capoccia, M.A. Riva, F. Cirulli, Decreased Bdnf expression and reduced social behavior in periadolescent rats following prenatal stress, Developmental psychobiology, 57 (2015) 365-373.

[9] V. Nieratschker, R. Massart, M. Gilles, A. Luoni, M.J. Suderman, B. Krumm, S. Meier, S.H. Witt, M.M. Nothen, S.J. Suomi, V. Peus, B. Scharnholz, H. Dukal, C. Hohmeyer, I.A. Wolf, F. Cirulli, P. Gass, M.W. Sutterlin, B. Filsinger, M. Laucht, M.A. Riva, M. Rietschel, M. Deuschle, M. Szyf, MORC1 exhibits cross-species differential methylation in association with early life stress as well as genome-wide association with MDD, Translational psychiatry, 4 (2014) e429.

[10] P. Pechtel, D.A. Pizzagalli, Effects of early life stress on cognitive and affective function: an integrated review of human literature, Psychopharmacology, 214 (2011) 55-70.

[11] E.R. De Kloet, E. Vreugdenhil, M.S. Oitzl, M. Joels, Brain corticosteroid receptor balance in health and disease, Endocrine reviews, 19 (1998) 269-301.

[12] F. Holsboer, The corticosteroid receptor hypothesis of depression, Neuropsychopharmacology, 23 (2000) 477-501.

[13] M. Joels, T.Z. Baram, The neuro-symphony of stress, Nature reviews. Neuroscience, 10 (2009) 459-466.

[14] A.B. Hittelman, D. Burakov, J.A. Iniguez-Lluhi, L.P. Freedman, M.J. Garabedian, Differential regulation of glucocorticoid receptor transcriptional activation via AF-1associated proteins, The EMBO journal, 18 (1999) 5380-5388.

[15] B.S. McEwen, J.H. Morrison, The brain on stress: vulnerability and plasticity of the prefrontal cortex over the life course, Neuron, 79 (2013) 16-29.

[16] S. Ridder, S. Chourbaji, R. Hellweg, A. Urani, C. Zacher, W. Schmid, M. Zink, H. Hortnagl, H. Flor, F.A. Henn, G. Schutz, P. Gass, Mice with genetically altered glucocorticoid receptor expression show altered sensitivity for stress-induced depressive reactions, J Neurosci., 25 (2005) 6243-6250.

[17] M.P. Boyle, J.A. Brewer, M. Funatsu, D.F. Wozniak, J.Z. Tsien, Y. Izumi, L.J. Muglia, Acquired deficit of forebrain glucocorticoid receptor produces depression-like changes in adrenal axis regulation and behavior, Proc Natl Acad Sci U S A, 102 (2005) 473-478.

[18] P. Gass, H.M. Reichardt, T. Strekalova, F. Henn, F. Tronche, Mice with targeted mutations of glucocorticoid and mineralocorticoid receptors: models for depression and anxiety?, Physiology \& behavior, 73 (2001) 811-825. 
[19] A. Urani, S. Chourbaji, P. Gass, Mutant mouse models of depression: candidate genes and current mouse lines, Neurosci Biobehav Rev, 29 (2005) 805-828.

[20] Q. Wei, X.Y. Lu, L. Liu, G. Schafer, K.R. Shieh, S. Burke, T.E. Robinson, S.J. Watson, A.F. Seasholtz, H. Akil, Glucocorticoid receptor overexpression in forebrain: a mouse model of increased emotional lability, Proc Natl Acad Sci U S A, 101 (2004) 11851-11856.

[21] C. Heim, D.J. Newport, S. Heit, Y.P. Graham, M. Wilcox, R. Bonsall, A.H. Miller, C.B. Nemeroff, Pituitary-adrenal and autonomic responses to stress in women after sexual and physical abuse in childhood, Jama, 284 (2000) 592-597.

[22] M.H. Teicher, A. Tomoda, S.L. Andersen, Neurobiological consequences of early stress and childhood maltreatment: are results from human and animal studies comparable?, Ann $\mathrm{N} \mathrm{Y}$ Acad Sci, 1071 (2006) 313-323.

[23] C. Caldji, B. Tannenbaum, S. Sharma, D. Francis, P.M. Plotsky, M.J. Meaney, Maternal care during infancy regulates the development of neural systems mediating the expression of fearfulness in the rat, Proc Natl Acad Sci U S A, 95 (1998) 5335-5340.

[24] D. Liu, J. Diorio, B. Tannenbaum, C. Caldji, D. Francis, A. Freedman, S. Sharma, D. Pearson, P.M. Plotsky, M.J. Meaney, Maternal care, hippocampal glucocorticoid receptors, and hypothalamic-pituitary-adrenal responses to stress, Science, 277 (1997) 1659-1662.

[25] I.C. Weaver, N. Cervoni, F.A. Champagne, A.C. D'Alessio, S. Sharma, J.R. Seckl, S. Dymov, M. Szyf, M.J. Meaney, Epigenetic programming by maternal behavior, Nat Neurosci, 7 (2004) 847-854.

[26] P.O. McGowan, A. Sasaki, A.C. D'Alessio, S. Dymov, B. Labonte, M. Szyf, G. Turecki, M.J. Meaney, Epigenetic regulation of the glucocorticoid receptor in human brain associates with childhood abuse, Nat Neurosci, 12 (2009) 342-348.

[27] C. Jensen Pena, C. Monk, F.A. Champagne, Epigenetic effects of prenatal stress on 11 beta-hydroxysteroid dehydrogenase- 2 in the placenta and fetal brain, PloS one, 7 (2012) e39791.

[28] Y. Li, P. Gonzalez, L. Zhang, Fetal stress and programming of hypoxic/ischemic-sensitive phenotype in the neonatal brain: mechanisms and possible interventions, Progress in neurobiology, 98 (2012) 145-165.

[29] B. Marciniak, J. Patro-Malysza, E. Poniedzialek-Czajkowska, Z. Kimber-Trojnar, B. Leszczynska-Gorzelak, J. Oleszczuk, Glucocorticoids in pregnancy, Current pharmaceutical biotechnology, 12 (2011) 750-757.

[30] C.J. Marsit, M.A. Maccani, J.F. Padbury, B.M. Lester, Placental 11-beta hydroxysteroid dehydrogenase methylation is associated with newborn growth and a measure of neurobehavioral outcome, PloS one, 7 (2012) e33794.

[31] S. Maccari, M. Darnaudery, S. Morley-Fletcher, A.R. Zuena, C. Cinque, O. Van Reeth, Prenatal stress and long-term consequences: implications of glucocorticoid hormones, Neurosci Biobehav Rev, 27 (2003) 119-127.

[32] S. Maccari, S. Morley-Fletcher, Effects of prenatal restraint stress on the hypothalamuspituitary-adrenal axis and related behavioural and neurobiological alterations, Psychoneuroendocrinology, 32 Suppl 1 (2007) S10-15.

[33] M. Kalinichev, K.W. Easterling, S.G. Holtzman, Early neonatal experience of Long-Evans rats results in long-lasting changes in reactivity to a novel environment and morphineinduced sensitization and tolerance, Neuropsychopharmacology, 27 (2002) 518-533.

[34] C.O. Ladd, R.L. Huot, K.V. Thrivikraman, C.B. Nemeroff, P.M. Plotsky, Long-term adaptations in glucocorticoid receptor and mineralocorticoid receptor mRNA and negative feedback on the hypothalamo-pituitary-adrenal axis following neonatal maternal separation, Biol Psychiatry, 55 (2004) 367-375.

[35] D. Liu, C. Caldji, S. Sharma, P.M. Plotsky, M.J. Meaney, Influence of neonatal rearing conditions on stress-induced adrenocorticotropin responses and norepinepherine release in the hypothalamic paraventricular nucleus, J Neuroendocrinol, 12 (2000) 5-12. 
[36] S.J. Suomi, Risk, resilience, and gene $x$ environment interactions in rhesus monkeys, Ann $\mathrm{N}$ Y Acad Sci, 1094 (2006) 52-62.

[37] E.B. Binder, D. Salyakina, P. Lichtner, G.M. Wochnik, M. Ising, B. Putz, S. Papiol, S. Seaman, S. Lucae, M.A. Kohli, T. Nickel, H.E. Kunzel, B. Fuchs, M. Majer, A. Pfennig, N. Kern, J. Brunner, S. Modell, T. Baghai, T. Deiml, P. Zill, B. Bondy, R. Rupprecht, T. Messer, O. Kohnlein, H. Dabitz, T. Bruckl, N. Muller, H. Pfister, R. Lieb, J.C. Mueller, E. Lohmussaar, T.M. Strom, T. Bettecken, T. Meitinger, M. Uhr, T. Rein, F. Holsboer, B. Muller-Myhsok, Polymorphisms in FKBP5 are associated with increased recurrence of depressive episodes and rapid response to antidepressant treatment, Nature genetics, 36 (2004) 1319-1325.

[38] E.B. Binder, The role of FKBP5, a co-chaperone of the glucocorticoid receptor in the pathogenesis and therapy of affective and anxiety disorders, Psychoneuroendocrinology, 34 Suppl 1 (2009) S186-195.

[39] E.B. Binder, R.G. Bradley, W. Liu, M.P. Epstein, T.C. Deveau, K.B. Mercer, Y. Tang, C.F. Gillespie, C.M. Heim, C.B. Nemeroff, A.C. Schwartz, J.F. Cubells, K.J. Ressler, Association of FKBP5 polymorphisms and childhood abuse with risk of posttraumatic stress disorder symptoms in adults, Jama, 299 (2008) 1291-1305.

[40] T. Klengel, D. Mehta, C. Anacker, M. Rex-Haffner, J.C. Pruessner, C.M. Pariante, T.W. Pace, K.B. Mercer, H.S. Mayberg, B. Bradley, C.B. Nemeroff, F. Holsboer, C.M. Heim, K.J. Ressler, T. Rein, E.B. Binder, Allele-specific FKBP5 DNA demethylation mediates genechildhood trauma interactions, Nat Neurosci, 16 (2013) 33-41.

[41] G. Guidotti, F. Calabrese, C. Anacker, G. Racagni, C.M. Pariante, M.A. Riva, Glucocorticoid receptor and FKBP5 expression is altered following exposure to chronic stress: modulation by antidepressant treatment, Neuropsychopharmacology, 38 (2013) 616-627.

[42] C. Anacker, P.A. Zunszain, A. Cattaneo, L.A. Carvalho, M.J. Garabedian, S. Thuret, J. Price, C.M. Pariante, Antidepressants increase human hippocampal neurogenesis by activating the glucocorticoid receptor, Molecular psychiatry, 16 (2011) 738-750.

[43] C.M. Pariante, R.B. Kim, A. Makoff, R.W. Kerwin, Antidepressant fluoxetine enhances glucocorticoid receptor function in vitro by modulating membrane steroid transporters, British journal of pharmacology, 139 (2003) 1111-1118.

[44] W. Chen, T. Dang, R.D. Blind, Z. Wang, C.N. Cavasotto, A.B. Hittelman, I. Rogatsky, S.K. Logan, M.J. Garabedian, Glucocorticoid receptor phosphorylation differentially affects target gene expression, Molecular endocrinology (Baltimore, Md.), 22 (2008) 1754-1766.

[45] N. Ismaili, M.J. Garabedian, Modulation of glucocorticoid receptor function via phosphorylation, Ann N Y Acad Sci, 1024 (2004) 86-101.

[46] R.S. Duman, L.M. Monteggia, A neurotrophic model for stress-related mood disorders, Biol Psychiatry, 59 (2006) 1116-1127.

[47] Y. Dwivedi, H.S. Rizavi, R.R. Conley, R.C. Roberts, C.A. Tamminga, G.N. Pandey, Altered gene expression of brain-derived neurotrophic factor and receptor tyrosine kinase $B$ in postmortem brain of suicide subjects, Arch Gen Psychiatry, 60 (2003) 804-815.

[48] J.R. Homberg, R. Molteni, F. Calabrese, M.A. Riva, The serotonin-BDNF duo: developmental implications for the vulnerability to psychopathology, Neurosci Biobehav Rev, 43 (2014) 35-47.

[49] F. Calabrese, R.H. van der Doelen, G. Guidotti, G. Racagni, T. Kozicz, J.R. Homberg, M.A. Riva, Exposure to early life stress regulates Bdnf expression in SERT mutant rats in an anatomically selective fashion, Journal of neurochemistry, 132 (2015) 146-154.

[50] F. Fumagalli, F. Bedogni, J. Perez, G. Racagni, M.A. Riva, Corticostriatal brain-derived neurotrophic factor dysregulation in adult rats following prenatal stress, The European journal of neuroscience, 20 (2004) 1348-1354. 
[51] A. Luoni, S. Hulsken, G. Cazzaniga, G. Racagni, J.R. Homberg, M.A. Riva, Behavioural and neuroplastic properties of chronic lurasidone treatment in serotonin transporter knockout rats, The international journal of neuropsychopharmacology, 16 (2013) 1319-1330.

[52] M. Roceri, F. Cirulli, C. Pessina, P. Peretto, G. Racagni, M.A. Riva, Postnatal repeated maternal deprivation produces age-dependent changes of brain-derived neurotrophic factor expression in selected rat brain regions, Biol Psychiatry, 55 (2004) 708-714.

[53] W.M. Lambert, C.F. Xu, T.A. Neubert, M.V. Chao, M.J. Garabedian, F.D. Jeanneteau, Brain-derived neurotrophic factor signaling rewrites the glucocorticoid transcriptome via glucocorticoid receptor phosphorylation, Molecular and cellular biology, 33 (2013) 37003714.

[54] M.J. Kempton, Z. Salvador, M.R. Munafo, J.R. Geddes, A. Simmons, S. Frangou, S.C. Williams, Structural neuroimaging studies in major depressive disorder. Meta-analysis and comparison with bipolar disorder, Arch Gen Psychiatry, 68 (2011) 675-690.

[55] K. Kingwell, Depression: In pursuit of happiness, Nature reviews. Drug discovery, 9 (2010) 918-919.

[56] P.J. Lucassen, O.J. Bosch, E. Jousma, S.A. Kromer, R. Andrew, J.R. Seckl, I.D. Neumann, Prenatal stress reduces postnatal neurogenesis in rats selectively bred for high, but not low, anxiety: possible key role of placental 11 beta-hydroxysteroid dehydrogenase type 2 , The European journal of neuroscience, 29 (2009) 97-103.

[57] J.S. Snyder, A. Soumier, M. Brewer, J. Pickel, H.A. Cameron, Adult hippocampal neurogenesis buffers stress responses and depressive behaviour, Nature, 476 (2011) 458461.

[58] T.J. Schoenfeld, H.A. Cameron, Adult neurogenesis and mental illness, Neuropsychopharmacology, 40 (2015) 113-128.

[59] T.J. Schoenfeld, E. Gould, Stress, stress hormones, and adult neurogenesis, Experimental neurology, 233 (2012) 12-21.

[60] M. Egeland, P.A. Zunszain, C.M. Pariante, Molecular mechanisms in the regulation of adult neurogenesis during stress, Nature reviews. Neuroscience, 16 (2015) 189-200.

[61] C. Anacker, A. Cattaneo, K. Musaelyan, P.A. Zunszain, M. Horowitz, R. Molteni, A. Luoni, F. Calabrese, K. Tansey, M. Gennarelli, S. Thuret, J. Price, R. Uher, M.A. Riva, C.M. Pariante, Role for the kinase SGK1 in stress, depression, and glucocorticoid effects on hippocampal neurogenesis, Proc Natl Acad Sci U S A, 110 (2013) 8708-8713.

[62] A. Bhargava, M.J. Fullerton, K. Myles, T.M. Purdy, J.W. Funder, D. Pearce, T.J. Cole, The serum- and glucocorticoid-induced kinase is a physiological mediator of aldosterone action, Endocrinology, 142 (2001) 1587-1594.

[63] M.L. Leong, A.C. Maiyar, B. Kim, B.A. O'Keeffe, G.L. Firestone, Expression of the serumand glucocorticoid-inducible protein kinase, Sgk, is a cell survival response to multiple types of environmental stress stimuli in mammary epithelial cells, The Journal of biological chemistry, 278 (2003) 5871-5882.

[64] C.A. Mikosz, D.R. Brickley, M.S. Sharkey, T.W. Moran, S.D. Conzen, Glucocorticoid receptor-mediated protection from apoptosis is associated with induction of the serine/threonine survival kinase gene, sgk-1, The Journal of biological chemistry, 276 (2001) 16649-16654.

[65] E.Y. Yuen, W. Liu, I.N. Karatsoreos, Y. Ren, J. Feng, B.S. McEwen, Z. Yan, Mechanisms for acute stress-induced enhancement of glutamatergic transmission and working memory, Molecular psychiatry, 16 (2011) 156-170.

[66] S. Miyata, Y. Koyama, K. Takemoto, K. Yoshikawa, T. Ishikawa, M. Taniguchi, K. Inoue, M. Aoki, O. Hori, T. Katayama, M. Tohyama, Plasma corticosterone activates SGK1 and induces morphological changes in oligodendrocytes in corpus callosum, PloS one, 6 (2011) e19859.

[67] A. Cattaneo, M. Gennarelli, R. Uher, G. Breen, A. Farmer, K.J. Aitchison, I.W. Craig, C. Anacker, P.A. Zunsztain, P. McGuffin, C.M. Pariante, Candidate genes expression profile 
associated with antidepressants response in the GENDEP study: differentiating between baseline 'predictors' and longitudinal 'targets', Neuropsychopharmacology, 38 (2013) 377 385.

[68] S. Miyata, T. Hattori, Disturbance of oligodendrocyte function plays a key role in the pathogenesis of schizophrenia and major depressive disorder, BioMed research international, in press (2015).

[69] [69] T. Frodl, F. Amico, Is there an association between peripheral immune markers and structural/functional neuroimaging findings?, Progress in neuro-psychopharmacology \& biological psychiatry, 48 (2014) 295-303.

[70] [70] T. Frodl, A. Carballedo, M.M. Hughes, K. Saleh, A. Fagan, N. Skokauskas, D.M. McLoughlin, J. Meaney, V. O'Keane, T.J. Connor, Reduced expression of glucocorticoidinducible genes GILZ and SGK-1: high IL-6 levels are associated with reduced hippocampal volumes in major depressive disorder, Translational psychiatry, 2 (2012) e88.

[71] A. Cattaneo, F. Macchi, G. Plazzotta, B. Veronica, L. Bocchio-Chiavetto, M.A. Riva, C.M. Pariante, Inflammation and neuronal plasticity: a link between childhood trauma and depression pathogenesis, Frontiers in cellular neuroscience, 9 (2015) 40.

[72] A. Luoni, F. Macchi, M. Papp, R. Molteni, M.A. Riva, Lurasidone exerts antidepressant properties in the chronic mild stress model through the regulation of synaptic and neuroplastic mechanisms in the rat prefrontal cortex, The international journal of neuropsychopharmacology, 18 (2015).

[73] K.A. Gaali S, Cuboni S, Hartmann J, Kozany C, Balsevich G, Namendorf C, FernandezVizarra P, Sippel C, Zannas AS, Draenert R, Binder EB, Almeida OF, Rühter G, Uhr M, Schmidt MV, Touma C, Bracher A, Hausch F., Selective inhibitors of the FK506-binding protein 51 by induced fit., Nature chemical biology, 11 (2015) 33-37. 
Figure 1

Schematic drawing of glucocorticoid receptor signaling cascade modulating FKBP5 and SGK1 function.

Glucocorticoid receptor (GR) is kept in the cytosol under an inactive form by its binding with the co-chaperonine FKBP5. When glucocorticoids (GC) bind GR, FKBP5 is exchanged against another co-chaperonine, FKBP4, which promotes the activation of GR that can be phosphorylated by several kinases (including the Serum Glucocorticoid Kinase 1, SGK1). GR can form dimers and thus translocates into the nucleus, where it binds Glucocorticoid Responsive Element (GRE) sequences on the DNA, activating the transcription of GR responsive genes, including FKBP5 and SGK1.

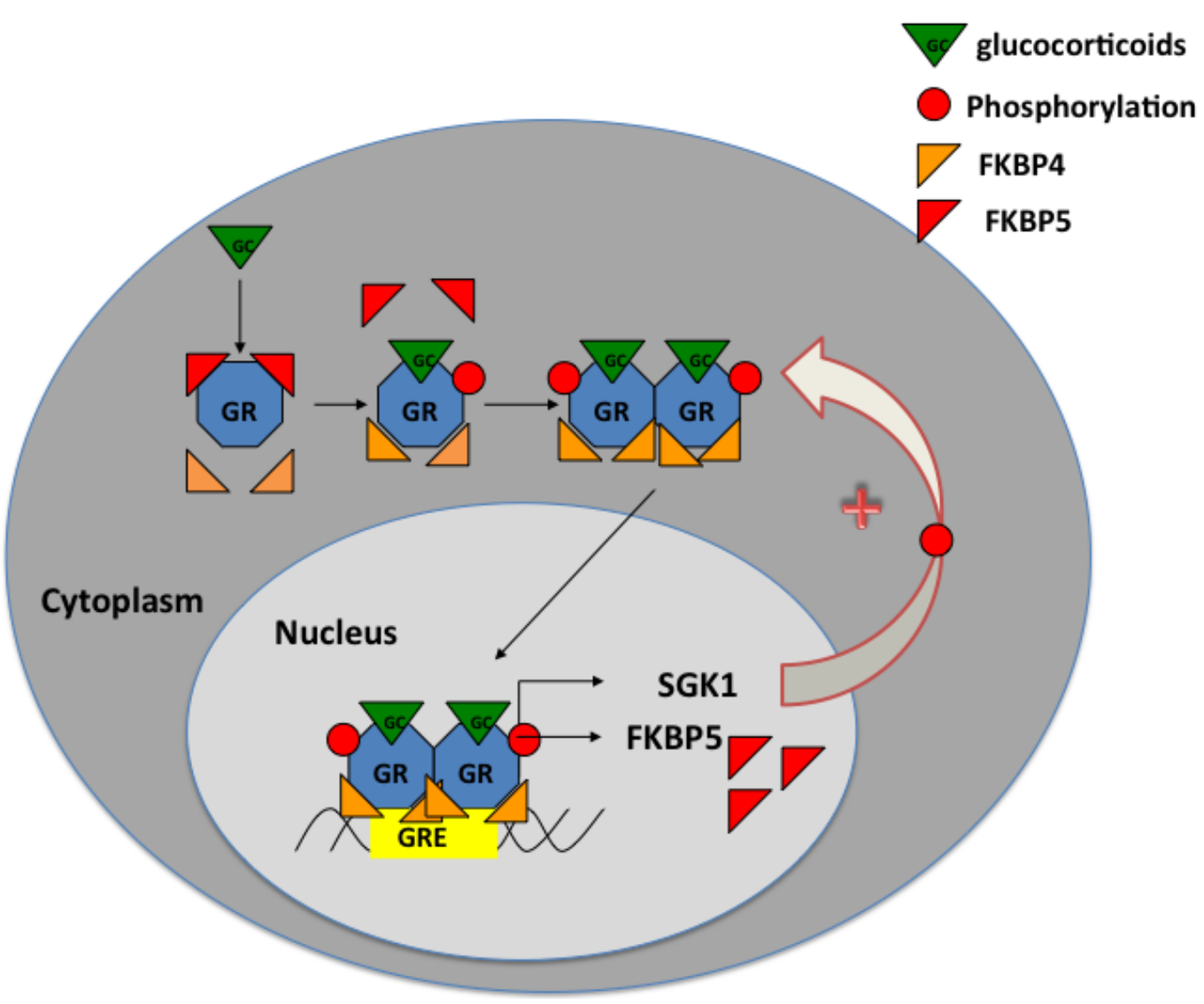




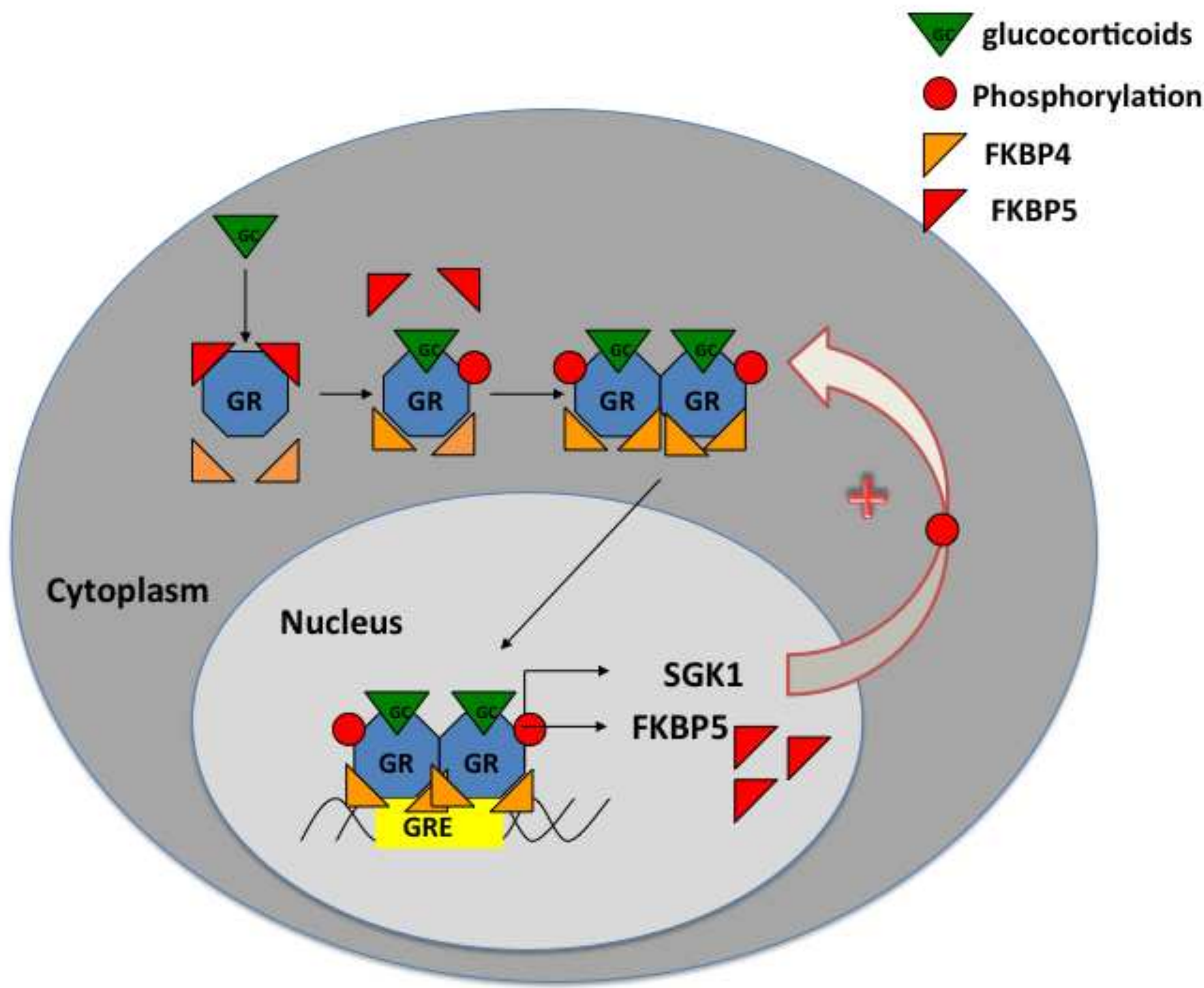

